\title{
The tumor inflammation signature (TIS) is associated with anti-PD-1 treatment benefit in the CERTIM pan-cancer cohort
}

Diane Damotte ${ }^{1,2,3,4}$, Sarah Warren ${ }^{5}$, Jennifer Arrondeau 4,6, Pascaline Boudou-Rouquette ${ }^{4,6}$, Audrey Mansuet-Lupo 1,2,3,4, Jérôme Biton ${ }^{7}$, Hanane Ouakrim,2,3, Marco Alifano 1,2,4, ${ }^{1}$, Claire Gervais ${ }^{4,6}$, Audrey Bellesoeur ${ }^{4,6}$, Nora Kramkimel $^{4,9}$, Camille Tlemsani ${ }^{4,6}$, Barbara Burroni ${ }^{3}$, Angéline Duche ${ }^{10}$, Franck Letourneur ${ }^{10}$, Han Si ${ }^{11}$, Rebecca Halpin ${ }^{11}$, Todd Creasy ${ }^{11}$, Ronald Herbst ${ }^{11}$, Xing Ren ${ }^{5}$, Pascale Morel ${ }^{5}$, Alessandra Cesano ${ }^{5}$, François Goldwasser ${ }^{2,4,6}$ and Karen Leroy ${ }^{2,4,12^{*}}$ (i)

\begin{abstract}
Background: The 18-gene tumor inflammation signature (TIS) is a clinical research assay that enriches for clinical benefit to immune checkpoint blockade. We evaluated its ability to predict clinical benefit of immunotherapy in cancer patients treated with PD-1 checkpoint inhibitors in routine clinical care.

Methods: The CERTIM cohort is a prospective cohort which includes patients receiving immune checkpoint inhibitors in Cochin University hospital. RNA extracted from 58 archival formalin fixed paraffin embedded tumor blocks (including 38 lung cancers, 5 melanomas, 10 renal carcinomas, 4 urothelial carcinomas and 1 colon carcinoma) was hybridized to a beta version of the NanoString ${ }^{\circledR}$ PanCancer $10360^{\mathrm{TM}}$ CodeSet using nCounter ${ }^{\circledR}$ technology. Gene expression signatures were correlated with tumor responses (by RECIST criteria) and overall survival. PD-L1 immunostaining on tumor cells was assessed in 37 non-small cell lung cancer (NSCLC) samples and tumor mutational burden (TMB) measured by whole exome sequencing in 19 of these.
\end{abstract}

Results: TIS scores were significantly associated with complete or partial response to anti-PD-1 treatment in the whole cohort (odds ratio $=2.64,95 \% \mathrm{Cl}[1.4 ; 6.0], p=0.008$ ), as well as in the NSCLC population (odds ratio $=3.27,95 \%$ $\mathrm{CI}[1.2 ; 11.6], p=0.03$ ). Patients whose tumor had a high TIS score (upper tertile) showed prolonged overall survival compared to patients whose tumor had lower TIS scores, both in the whole cohort (hazard ratio $=0.37,95 \% \mathrm{Cl}[0.18$, $0.76], p=0.005$ ) and in the NSCLC population (hazard ratio $=0.36,95 \% \mathrm{Cl}[0.14,0.90], p=0.02$ ). In the latter, the TIS score was independent from either PD-L1 staining on tumor cells (spearman coefficient 0.2) and TMB (spearman coefficient - 0.2).

Conclusions: These results indicate that validated gene expression assay measuring the level of tumor microenvironment inflammation such as TIS, are accurate and independent predictive biomarkers and can be easily implemented in the clinical practice.

Keywords: Immunotherapy, PD-1, Interferon, Tumor, Inflammation, Signature

\footnotetext{
*Correspondence: karen.leroy@aphp.fr

${ }^{12}$ Department of Genetic and Molecular Biology, Hôpital Cochin, AP-HP,

27 rue du Faubourg St Jacques, 75014 Paris, France

Full list of author information is available at the end of the article
} 


\section{Background}

Immune checkpoint inhibitors (ICI), specifically blockade of the PD-1/PD-L1 pathway via monoclonal antibodies, have entered the oncology therapeutic arsenal and are being evaluated in an increasing number of indications, although the response rates as single agents and in unselected patient populations are usually low. A variety of biomarkers are currently being explored as strategies to enrich for clinical responders, and for some indications, approvals of the anti-PD-1/PD-L1 therapeutic incorporate biomarker testing in the form of PD-L1 measurement by immunohistochemistry (IHC) into the prescribing label. Despite being cleared by the FDA as companion and/or complementary diagnostics for different anti-PD-1/PD-L1 drugs, PD-L1 IHC assays have a number of limitations, including suboptimal positive and negative predictive value and reproducibility. As ICI disrupt pathways that are conserved between tumor cells from multiple different tumor types and the immune environment, biomarkers which are predictive across a variety of indications are of particular interest.

Several biomarkers have been developed which characterize either the potential for tumor cells to prime an adaptive immune response, e.g. tumor mutation burden (TMB) and microsatellite instability (MSI), or the downstream consequences of immune activation, as measured by presence of immune cells e.g. Immunoscore, or gene expression signature related to immune environment [1]. A number of candidate gene expression signatures have been developed, and most focus on biology of activated $\mathrm{T}$ cells and IFNY signaling [2,3] Interestingly, defects in the IFN $\gamma$ signaling pathway have been identified as a mechanism of resistance, strongly supporting a crucial role of IFN $\gamma$ in the anti-cancer immune response $[4,5]$. An association between IFN $\gamma$ expression or IFN $\gamma$ inducible gene signature and clinical responses to anti-PD-1/ PD-L1 mAb was reported in melanoma [6] and bladder carcinomas [7]. Recently an 18 gene "tumor inflammation signature" (TIS) which quantifies an activated but suppressed adaptive immune response in the tumor microenvironment, was demonstrated to retrospectively predict clinical benefit of anti-PD-1 in various cancer types (melanoma, head and neck squamous cell carcinomas, digestive cancers, ovarian and triple negative breast cancers) in clinical trials [2]. The signature has been analytically validated $[8,9]$ and is currently under investigation in multiple Research Use Only (RUO) and Investigational Use Only (IUO) studies for performance as a predictive biomarker.

In order to assess the feasibility and utility of gene expression profiling in routine clinical practice, we assessed the TIS score from a mixed tumor cohort of patients treated with anti-PD-1 on label at a single center as part of a larger transcriptional profiling study with the PanCancer IO360 gene expression panel.

\section{Methods \\ Patients}

Fifty-eight patients from the CERTIM (Immunomodulatory Therapies Multidisciplinary Study group) were included in the study. The CERTIM cohort was initiated in our hospital in July 2015 and prospectively includes all the patients treated with a PD-1 checkpoint inhibitor (either nivolumab or pembrolizumab) administered per label as a single agent, for advanced solid tumor. Treatment was continued until disease progression or intolerable toxicity, physician or patient decision. Tumor imaging by $\mathrm{CT}$ scan was performed at baseline, every 8 weeks for patients receiving nivolumab and every 6 weeks for patients receiving pembrolizumab through the first 6 months and every 12 weeks for both thereafter. Response was assessed per Response Evaluation Criteria in Solid Tumors (RECIST) version 1.1. Clinically stable patients considered to be deriving clinical benefit could continue therapy until disease progression was confirmed on imaging done at least 4 weeks after the first assessment of stable disease. The toxicities observed in the patient cohort were monthly reviewed by the dedicated CERTIM multidisciplinary board. Clinical status was assessed monthly during treatment and continued when the treatment was stopped. Database lock-up was 9 February 2018. Median patients follow-up was 19.2 months.

This study was approved by the ethics committee (CPP Ile de France II, no. 2008-133, 2012 06-12, 2018 MS1) in agreement with article L.1121-1 of French law.

\section{PD-L1 immunostaining}

For each tumor, we performed PD-L1 immunostaining on fresh-cut slides from representative FFPE blocks using an anti-PD-L1 antibody (E1L3N, Cell signaling) on Bond automat (Leica) as previously described and validated by the PATTERN French thoracic pathologists group [10]. Staining was blinded analyzed (DD, ALM, BB) on tumoral cells.

\section{Genomic DNA extraction and illumina-based whole-exome sequencing}

Genomic DNA from 22 tumors was isolated from formalin-fixed paraffin-embedded (FFPE) blocks using Maxwell 16 FFPE Tissue LEV DNA Purification Kit (Promega), according to the manufacturer's instructions. Wholeexome was sequenced as previously reported [11]. DNA were sequenced on the Novaseq 6000 platform, using at least $100 \mathrm{ng}$ of double stranded tumoral DNA (Qubit dsDNA HS kit). After shearing, 11 randomly selected samples were run on the Agilent Bioanalyzer to confirm 
successful shearing prior to library construction The SureSelect Human All Exon V6 capture kit was used to capture coding regions of genes included in the major genomic databases. Paired end FASTQ files of $101 \mathrm{mer}$ sequence reads were generated. All sequence data was quality controlled for read counts, quality values, kmer usage, GC-content, and all other relevant parameters with FastQC (v0.10.1). The DNA read sequences were aligned to the genome (UCSC hg19; Feb 2009 release) using BWA (v0.7.15) and reads sorting and PCR duplicate removal were conducted using Picard (v2.8.3). VarScan2 (v2.4.2) with Samtools mpileup (v1.3) was used to call SNVs/Indels against human reference genome. Germline polymorphisms were removed by retaining only variants with MAF in all races of $<1 \%$ or unknown MAF within the 1000 genomes and NHLBI-ESP project with 6500 exome database. The retained SNVs/indels were further filtered by removing SNPs in dbSNP129. Depth of sequencing coverage was $60 \times$ on average across all samples in the cohort.

\section{RNA extraction and hybridization to nCounter codeset}

Archival biopsies of patients included in the CERTIM cohort, sampled before anti-PD-1 treatment and with adequate tumor tissue left in FFPE blocks were selected for RNA extraction. RNA was extracted with High Pure FFPE RNA Isolation Kit (Roche) from FFPE tumor samples according to the recommendations of the manufacturer and quantified using fluorimetry with $\mathrm{Qubit}^{\mathrm{TM}} \mathrm{RNA}$ XR Assay Kit (Invitrogen). 100 ng RNA (46 samples) or 30 to 85 ng RNA (12 samples) were successfully hybridized to a beta version of the NanoString ${ }^{\circledR}$ PanCancer IO 360 Panel code set, according to the recommendations of the manufacturer.

\section{Statistical analysis of transcriptional data}

Raw data for each sample and gene were normalized to internal ERCC controls to eliminate technical variability of the assay, and then counts were normalized to the geometric mean of endogenous housekeeping genes followed by $\log 2$ transformation. Gene expression signatures, including the TIS, were calculated as a weighted linear average of the constituent genes [2, 12]. The weighted scores used for calculation of the TIS and other signatures are NanoString intellectual property. For the correlation analysis with clinical response to treatment, clinical benefit was defined as complete or partial RECIST response while stable and progressive disease were defined as lack of clinical benefit. Normalized gene counts and signature scores were compared to the response category using a linear model. The $\log 2$ fold change, Wald-type confidence interval and $p$-value were calculated for each gene and signature (Additional file 1:
Table S1 and Additional file 2: Table S2). To assess predictive performance of the additional signatures above and beyond the predictive performance of TIS, a logistic regression model was used to assess performance conditional upon TIS score.

For the analysis on the survival time, the genes and scores were dichotomized into high and low groups based on median, with the exception of TIS which was divided into tertiles. The survival time was fit to the binary group with Cox proportional hazard model. The hazard ratio between the high and low group, the Waldtype confidence interval and the log-rank test $p$-value are reported for each gene and signature (Additional file 1: Table S1 and Additional file 2: Table S2).

\section{Results}

Tumor inflammation signature enriches for clinical response to anti-PD-1 in the multi-tumor cohort

Consecutive metastatic cancer patients treated with antiPD-1 monoclonal antibodies in the outpatient monocentric CERTIM cohort with available FFPE tumor specimen were selected for analysis of gene expression and immune signatures using NanoString PanCancer IO 360 Panel (beta version), which contains an RUO version of the TIS. The clinical characteristics of the 58 patients with different cancer types included in this study are described in Table 1. Several genes showed a differential expression in responders to anti-PD-1 [patients with complete response (CR) or partial response (PR) according to RECIST criteria] compared to non-responders (stable or progressive disease) (Additional file 1: Table S1). After correction for multiple testing, 5 genes related to IFNy signaling and antigen processing remained significantly higher in responders: CXCL9, CXL10, CXCL11, TAP1 and PSMB9 (Fig. 1a). Since a number of the genes with greatest association with clinical benefit are contained within or closely related to genes in the TIS, we evaluated the TIS as a predictive biomarker in this cohort. In this study, a high TIS score was significantly associated with response to anti-PD- 1 treatment (odds ratio $=2.64$, 95\% CI [1.4; 6.0], $p=0.008$, Logistic regression) (Fig. 1b). Furthermore, patients with a high TIS score (upper tertile) had a prolonged overall survival compared to patients with lower scores (hazard ratio $=0.37,95 \%$ CI $[0.18,0.76], p$-value $=0.005$, Cox regression) (Fig. 1c). The expression of 16 genes included in the TIS score were closely correlated, whereas $C D 276$ and $H L A-D Q A 1$ expression appeared more variable across TIS scores (Fig. 1d). The normalized gene expression data, TIS score, as well as response to ICI and survival for each of the samples included in this study are provided in Additional file 3: Table S3. Altogether, these data indicate that 
Table 1 Clinical characteristics of the patients in the CERTIM multi-cancer cohort

\begin{tabular}{lc}
\hline & $\boldsymbol{N}(\%)$ \\
\hline Sex & \\
M & $38(66 \%)$ \\
F & $20(44 \%)$ \\
Age (year) & \\
Median (range) & $66(41-83)$ \\
Tumor type & \\
Non-small cell lung & $37(64 \%)$ \\
Small cell lung & $1(2 \%)$ \\
Melanoma & $5(9 \%)$ \\
Colon & $1(2 \%)$ \\
Renal & $10(17 \%)$ \\
Urothelial & $4(7 \%)$ \\
ECOG performance status & \\
0 & \\
1 & $2(3 \%)$ \\
$\geq 2$ & $31(54 \%)$ \\
Previous lines of therapy & $24(42 \%)$ \\
0 & \\
1 & $2(3 \%)$ \\
2 & $31(53 \%)$ \\
Anti-PD-1 3 & $11(19 \%)$ \\
Nivolumab & $14(24 \%)$ \\
ORR & \\
PR & $52(90 \%)$ \\
PD & $6(10 \%)$ \\
\hline ECOG staizumab & $6(10 \%)$ \\
\hline
\end{tabular}

${ }^{a}$ ECOG status was not available for 1 patient

the TIS is significantly associated with clinical benefit of anti-PD-1 (pembrolizumab or nivolumab) in a «real life» cohort of patients.
TIS predictive of anti-PD-1 benefit in non small cell lung cancer (NSCLC) cohort

We then focused our analysis on NSCLC which represented the majority of the cases that were studied in this cohort. All 37 patients had received nivolumab, and the clinical characteristics of the patients, including the tumor subtype and smoking status, are indicated in Table 2. Overall, 7/37 (19\%) patients responded to treatment. As in the whole cohort, we observed that TIS enriched for tumor response in NSCLC (odds ratio $=3.27,95 \%$ CI $[1.2 ; 11.6], p=0.03$, Logistic regression) (Fig. 2a). Furthermore, patients with a high TIS score (upper tertile) had a prolonged survival compared to those with lower TIS scores (hazard ratio $=0.36,95 \%$ CI $[0.14,0.90], p$-value $=0.02$, Cox regression) (Fig. 2b). In order to assess the predictive value of TIS score within the context of one of other biomarkers of clinical interest, we also scored the NSCLC samples for PD-L1 expression by $\mathrm{IHC}$ at the $1 \%$ and $50 \%$ cutoff for tumor cell positivity, the two cut offs reported in the label for second and first line NSCLC patient selection for treatment with pembrolizumab. PD-L1 staining $\geq 1 \%$ tumor cells was not significantly associated with survival (hazard ratio $=0.87$, CI $[0.4,2], p=0.74)$, and PD-L1 staining on $50 \%$ tumor cells had a trend with overall survival but did not reach statistical significance (hazard ratio $=0.40, \mathrm{CI}[0.1,1.3]$, $p=0.13$ ) (Fig. 2c, d). Additionally, tumor mutational burden by whole exome sequencing was available for 19 of the patients (see Additional file 4: Table S4 for baseline characteristics). The tumor mutational burden ranged from 13.7 to 26.8 mutations/MB and was positively correlated with the number of smoking pack years (spearman coefficient 0.43 ). In this limited patient cohort, TMB was lower in adenocarcinoma $(n=11$, median $=20.3$ mutation $/ \mathrm{Mb})$ than in squamous cell carcinoma $(\mathrm{n}=6$, median $=23.3$ mutation $/ \mathrm{Mb})(p$ value $=0.01$, Fisher test $)$, and was not significantly associated with survival (hazard ratio $=1.91$, CI $[0.6,6.2], p=0.25)$. In this small cohort, TIS was still significantly associated with overall survival

\footnotetext{
(See figure on next page.)

Fig. 1 TIS scores are associated with response to anti-PD-1 in the CERTIM multi-tumor cohort. a Volcano plot of $p$-value versus log2 fold change of the differential expression between responders (PR/CR) and non-responders (PD/SD) in the whole cohort. The test for differential expression was done by fitting the log2 normalized count to the response with linear model. The $p$-values were adjusted by the Benjamini-Hochberg Procedure. Dots corresponding to genes that are significant at FDR $<0.1$ are labelled in red. $\mathbf{b}$ Boxplot of TIS scores in responders and non-responders (The lower whisker of the responders is not visible as the length is 0 ). The response was fit to TIS scores with logistic regression and $p$-value $=0.008$, indicating that high TIS scores are predictive of response to anti-PD-1 treatment. The odds ratio is $2.64,95 \%$ confidence interval $(1.37,5.95)$. c The Kaplan-Meier curves of TIS score groups for the whole patient cohort. The TIS scores were categorized into three groups by tertiles. The KaplanMeier curves show that the high TIS score group have higher survival rate than the other two groups (which are combined on the graph into the "low" group). The survival time was fit to TIS score group (high vs low and intermediate) with Cox proportional hazard model. Hazard ratio is 0.374 , $95 \%$ confidence interval $(0.18,0.76)$, $p$-value $=0.005$, meaning the high TIS score group has a decrease of the hazard by $63 \%$. $\mathbf{d}$ Heatmap showing the individual TIS genes normalized expression, as well as TIS global score, histological subtype and overall response to anti-PD1. NSCLC non small cell lung carcinoma, RCC renal cell carcinoma, SCLC small cell lung carcinoma, nivo nivolumab, pembro pembrolizumab, ORR overall response according to RECIST v1.1, CR complete response, PR partial response, SD stable disease, PD progressive disease
} 


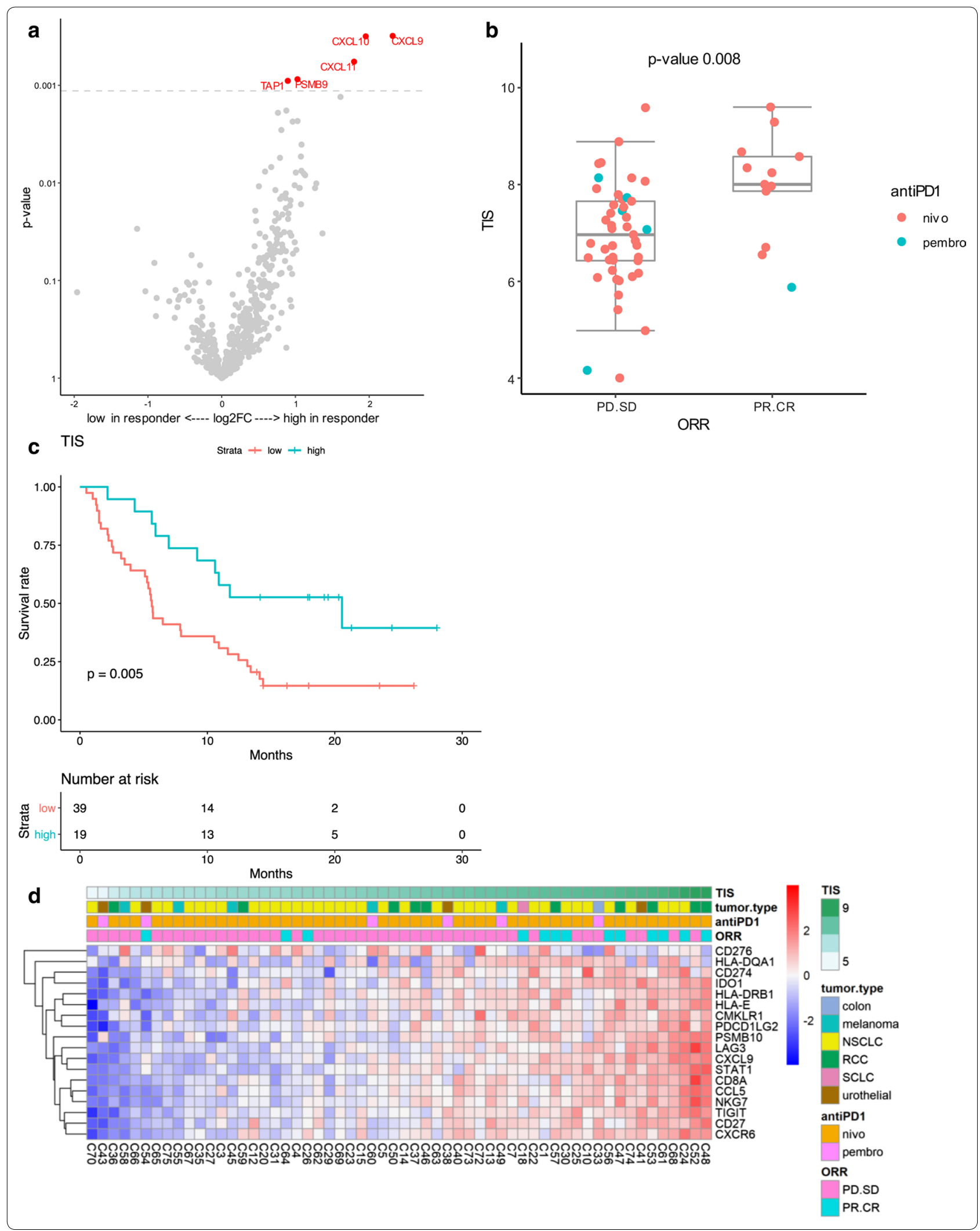




\begin{tabular}{|c|c|c|}
\hline Characteristic & Category & N (\%) \\
\hline \multirow[t]{2}{*}{ Sex } & M & $23(62 \%)$ \\
\hline & $\mathrm{F}$ & $14(38 \%)$ \\
\hline Age (year) & Median (range) & $68(41-78)$ \\
\hline \multirow[t]{3}{*}{ Tumor type } & Adenocarcinoma & $25(68 \%)$ \\
\hline & Squamous cell carcinoma & $10(27 \%)$ \\
\hline & NOS & $2(5 \%)$ \\
\hline \multirow[t]{7}{*}{ Smoking status } & Non smoker & $4(11 \%)$ \\
\hline & Smokers & $33(88 \%)$ \\
\hline & $\begin{array}{l}<10 \text { pack/year ( } \leq 10 \text { packs } \\
\text { years) }\end{array}$ & - \\
\hline & [10-30] pack/year & $19(57 \%)$ \\
\hline & > 30 pack/year & $14(42 \%)$ \\
\hline & Quit $>1$ year & $19(57 \%)$ \\
\hline & Active or quit $\leq 1$ year & $14(42 \%)$ \\
\hline \multirow[t]{3}{*}{ ECOG performance status } & 0 & $1(3 \%)$ \\
\hline & 1 & $18(49 \%)$ \\
\hline & $\geq 2$ & $18(49 \%)$ \\
\hline \multirow[t]{4}{*}{ Previous lines of therapy } & 0 & - \\
\hline & 1 & $22(59 \%)$ \\
\hline & 2 & $6(16 \%)$ \\
\hline & $\geq 3$ & $9(24 \%)$ \\
\hline \multirow[t]{4}{*}{ ORR } & $C R$ & $3(8 \%)$ \\
\hline & $P R$ & $4(11 \%)$ \\
\hline & SD & $6(16 \%)$ \\
\hline & PD & 24 (65\%) \\
\hline
\end{tabular}

$(\mathrm{p}=0.02$, data not shown). Finally, we assessed whether any of the biomarkers were associated with one another, and observed that PD-L1 staining on tumor cells and TMB were positively correlated with tobacco exposure, but the other biomarkers were not strongly associated with each other (Fig. 2e). Specifically, PD-L1 IHC staining was not significantly with TMB (spearman coefficient
-0.16 , $\mathrm{p}$ value 0.53 ), and the TIS was not significantly correlated with either PD-L1 immunohistochemical staining (spearman coefficient 0.20 , p value 0.25 ), or TMB (spearman coefficient -0.22 , p value 0.38 ).

\section{Additional signatures beyond TIS predictive of anti-PD-1 benefit}

We next evaluated a number of other predefined immune gene signatures contained within the IO360 panel for their association with response in the NSCLC cohort and identified several signatures that were statistically significant, including IFN $\gamma$ signaling, lymphoid cells, T cell, NK cells, cytotoxic cells, exhausted CD8 T cells, macrophages, stroma, inflammatory chemokines, and immunoproteasome (Fig. 3a). The majority had strong positive correlation with TIS and with each other (Fig. 3b). Multivariate analysis showed that none of these gene signatures significantly predicted for response after correcting for TIS (data not shown). The signatures were then evaluated to see which were also predictive of overall survival in the NSCLC cohort, and only TIS, stroma, and lymphoid signatures were significantly associated (Additional file 5: Figure S1). In the multi-tumor cohort, several signatures were associated with response, but only IFNy signature remained significant after adjusting for TIS ( $p$-value $=0.03$ ) (Additional file 6: Figure S2A); whereas TIS, immunoproteasome, lymphoid and hypoxia signatures were predictive of survival (Additional file 6: Figure S2B). These findings indicate that TIS is a robust biomarker of both response and survival following PD-1 checkpoint blockade in both NSCLC and multi-tumor cohorts from real world patients.

\section{Discussion}

The limited efficacy, potential toxicity and the cost of anti-PD-1/PD-L1 molecules in cancers patients calls for the use of predictive biomarkers. Our study focused on gene expression based biomarkers because they are

\footnotetext{
(See figure on next page.)

Fig. 2 Performance of TIS assay vs other biomarkers in NSCLC cohort. a Boxplot of TIS scores in responders and non-responders in the NSCLC cohort. All patients were treated with nivolumab. The response was fit to TIS scores with logistic regression and $p$-value $=0.033$, indicating that high TIS scores are predictive of tumor response to anti PD-1 treatment. The odds ratio is 3.27, 95\% confidence interval (1.23, 11.63). b The Kaplan-Meier curves of TIS score groups for the NSCLC cohort. Patients are stratified by TIS score tertiles, and the highest TIS score group was observed to have longer survival than the other two groups (which are combined into the "low" group on the graph). The survival time is fit to TIS score group (high vs low and intermediate) with Cox proportional hazard model. Hazard ratio is $0.36,95 \%$ confidence interval $(0.14,0.90)$, $p$-value $=0.0235$, meaning the high TIS score group has a decrease of the hazard by 64\%. c The Kaplan-Meier curves of NSCLC patients with $1 \%$ PD-L1 positivity on tumor cells used as a cutoff. The survival time in the NSCLC cohort is fit to PD-L1+ group (high vs low) with Cox proportional hazard model. d The KaplanMeier curves of NSCLC patients with 50\% PD-L1 positivity on tumor cells used as a cutoff. The survival time in the NSCLC cohort is fit to PD-L1+ group (high vs low) with Cox proportional hazard model. Analysis suggests that patients with 50\% PD-L1+ tumor cells may have longer survival, but the sample size is too limited to reach statistical significance. e The Spearman correlation matrix between TIS scores, percentage of PD-L1+ tumor cells, tumor mutation burden and tobacco exposure for NSCLC cohort. PD-L 1+ tumor cells and TMB are positively correlated with tobacco exposure
} 


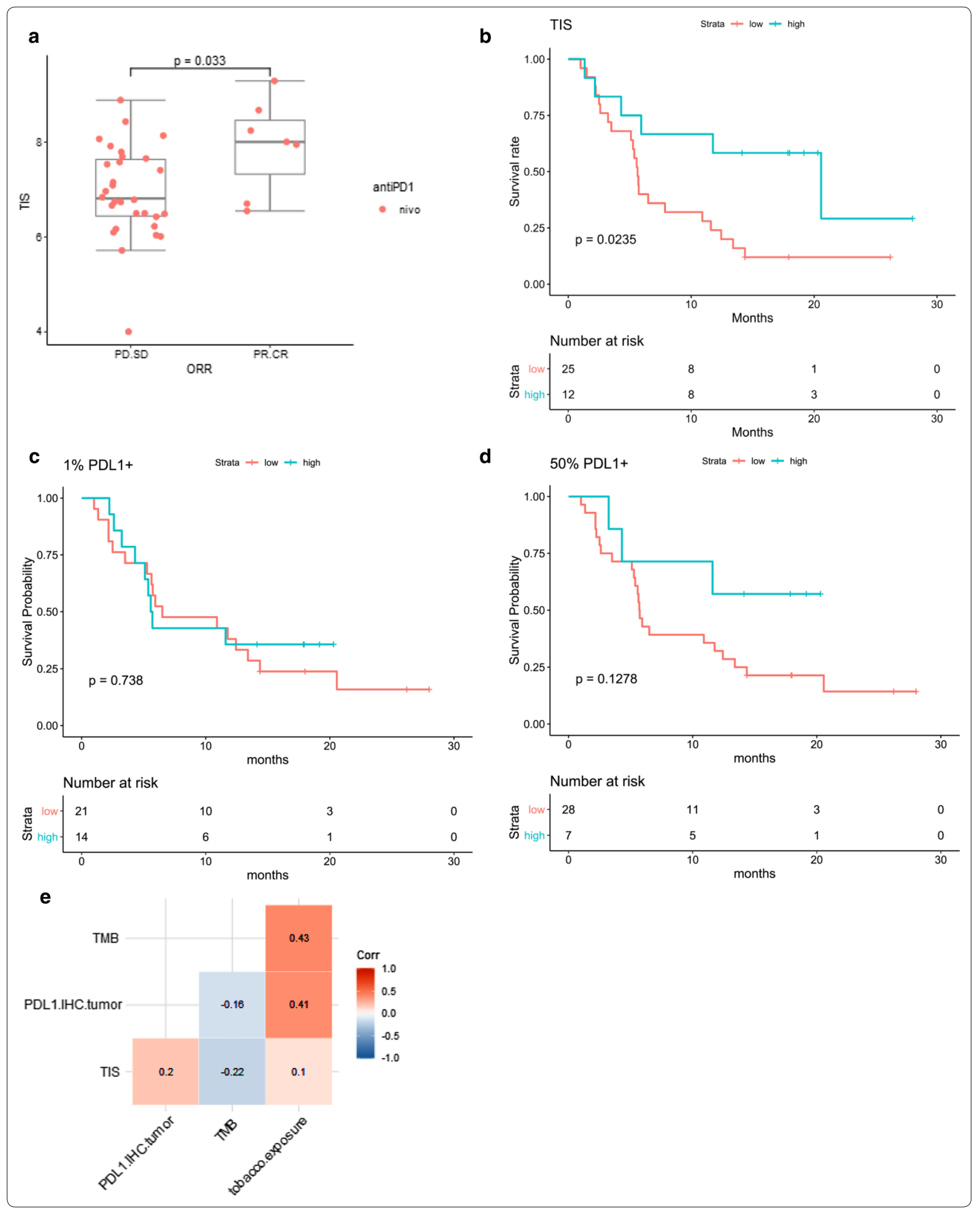




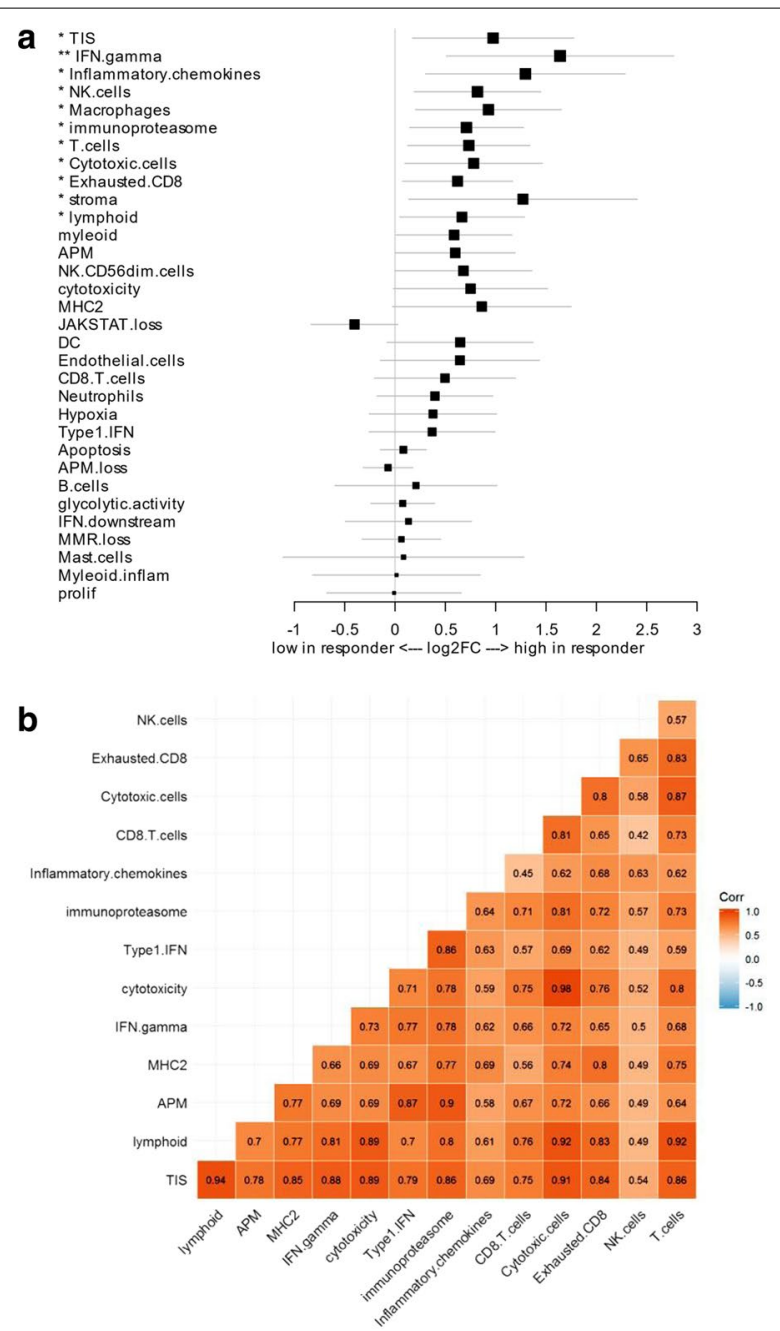

Fig. 3 Additional gene expression signatures associated with clinical benefit of anti-PD-1 in NSCLC. a Forest plot of difference of multi-gene signature scores between responders and non-responders in the NSCLC cohort. The position of the squared dots denotes the difference of score, and the size denotes the statistical significance. The horizontal lines are the Wald-type confidence intervals. The * sign denotes the significance of $p$-value $\left(<0.001^{* * *},<0.01^{* *},<0.05^{*}\right)$. b The Spearman correlation matrix between the signature scores was calculated for the signatures that showed significant difference in the differential expression analysis between responder and non-responders

compatible with current clinical practice, i.e. they can be performed with small tissue samples and using a local laboratory and have the potential for clinical utility across disease indications and therapeutic agents. The TIS was originally described as a biomarker predictive of response in patients with different cancer types, treated with pembrolizumab in the context of clinical trials [2]. Here, we extend the characterization of TIS to include novel indications (NSCLC) and novel agents (nivolumab). One strength of this study is that it was performed on patients treated in the community setting; thus in a less selected patient population than in clinical trials and likely more reflective of larger real world performance. This study demonstrates an association of high TIS with ICI benefit, for both clinical response and overall survival, independently of carcinoma subtype and anti-PD-1 therapeutic molecule administered.

The study has a number of limitations which must be considered in the interpretation of results. First, the study is composed of a small cohort of patients, especially the lung cancer cohort. Despite the small cohort size, the TIS was predictive of both response and survival, and thus may represent a realistic option for the identification of patients who may benefit from ICI. We await the availability of larger cohorts of patients treated with ICI in the real life management to confirm these results. Ultimately, prospective clinical trials using TIS as a stratifying biomarker are needed to definitely confirm its predictive value in different cancer types.

It is worth considering the performance of the nongene signature biomarkers in this study. Although it is the approved diagnostic, PD-L1 IHC was not observed to be predictive, which may be due to tissue heterogeneity and the cohort size. Small biopsies sometimes comprise small numbers of tumor cells and the positivity of these cells may not reflect the whole tumor status [13]. Using multigene signatures such as the TIS may be a more robust way of measuring the presence of a cytotoxic anti-tumor immune response than measuring PD-L1 protein alone. Similarly, high TMB has been shown to be associated with ICI benefit, particularly in lung cancer, in numerous studies [14-17], but it was not predictive in this cohort of NSCLC, nor was it correlated with TIS. This may be due to the limited number of cases studied, mixed histology (adenocarcinoma and SCC) and/or the fact that TMB was high in all samples (13.7 to 26.8 mutations/MB). Interestingly, a recent study established that $\mathrm{TMB}$ and TIS were independently predictive of clinical response to pembrolizumab in KEYNOTE clinical trials in a variety of tumors but without examining NSCLC [18]. Further limiting its utility, at the current time TMB measurement is a costly technique, and is awaiting for international standardization and recommendations [19].

Finally, gene signatures beyond TIS may be required in the future to further dissect mechanisms of immune resistance in patients whose tumor does not respond to single agent PD-1/PD-L1 blockade to inform biologybased combinations. Specifically, while tumor inflammation gene signatures, including TIS, are measuring IFN biology and/or presence of specific $\mathrm{T}$ cell populations [2, $6,20-22]$, additional signatures (which could be combined with TIS) are needed to gain further insight into 
additional processes associated with immune escape that could be targeted therapeutically [23].

\section{Conclusions}

In this study, gene expression signatures were analyzed in a cohort of FFPE tumor samples from cancer patients treated with anti-PD-1 in routine clinical care. The tumor inflammation signature was significantly associated with clinical response and overall survival, supporting its evaluation in parallel with other biomarkers in routine practice and future clinical trials.

\section{Supplementary information}

Supplementary information accompanies this paper at https://doi. org/10.1186/s12967-019-2100-3.

Additional file 1: Table S1. Statistical correlation of individual gene expression with response and survival in the whole cohort and in the NSCLC cohort.

Additional file 2: Table S2. Statistical correlation of gene signatures with response and survival in the whole cohort and in the NSCLC cohort.

Additional file 3: Table S3. Histological subtype, clinical outcome sion data, for each of the 58 samples included in the study.

Additional file 4: Table S4. Clinical characteristics of the NSCLC patients

Additional file 5: Figure S1. Immune signatures associated with overall survival in the NSCLC cohort. Forest plot of hazard ratio in the survival analysis between high and low signature score in the NSCLC cohort. The signature scores are dichotomized into high and low groups by their median (except TIS scores uses the upper tertile as in Fig. 2b). The survival time is fit to score group (high vs low) with Cox proportional hazard model. The hazard ratio and Wald-type confidence interval are estimated. The $p$-value are determined by the log-rank test. The * sign denotes the significance of $p$-value $\left(<0.001^{* * *},<0.01^{* *},<0.05^{*}\right)$.

Additional file 6: Figure S2. TIS scores and other gene signatures associated with response and overall survival in the CERTIM multi-tumor cohort. a Forest plot of difference of multi-gene signature scores between responders and non-responders in the CERTIM multi-tumor cohort. The position of the squared dots denotes the difference of score, and the size denotes the statistical significance. The horizontal lines are the Waldtype confidence intervals. The * sign denotes the significance of $p$-value $\left(<0.001^{* * *},<0.01^{* *},<0.05^{*}\right)$. b Forest plot of hazard ratio in the survival analysis between high and low signature score in the CERTIM cohort. The signature scores are dichotomized into high and low groups by their median (except TIS scores uses the upper tertile as in Fig. 1c). The survival time is fit to score group (high vs low) with Cox proportional hazard model. The hazard ratio and Wald-type confidence interval are estimated. The $p$-value are determined by the log-rank test. The * sign denotes the significance of $p$-value $\left(<0.001^{* * *},<0.01^{* *},<0.05^{*}\right)$. (response to anti-PD1 and survival), TIS score and normalized gene expreswith TMB data.

\section{Authors' contributions}

$\mathrm{DD}, \mathrm{FG}$ and $\mathrm{KL}$ designed the study, analyzed and interpreted the data and prepared the manuscript. SW, XR, PM, and AC designed, analyzed, and interpreted the gene expression data; and prepared the manuscript. $\mathrm{RH}, \mathrm{HS}, \mathrm{RH}$ and TC provided the tumor mutation burden assessment. JA, PBR, CG, AB, NK, CT and MA provided clinical data. AML and BB performed PDL1 assessment. JB and $\mathrm{HO}$ performed RNA extraction. FL and AD run the nCounter assay. All authors read and approved the final manuscript.

\section{Funding}

This work was supported by the Institut National de la Santé et de la Recherche Médicale (INSERM); Paris Descartes-Paris 5 University; Pierre et Marie Curie-Paris 6 University; the Cancer Research for Personalized Medicine (CARPEM); the LabEx Immuno-oncology; ITMO Cancer, National Alliance for Life Sciences \& Health, within the framework of the Cancer Plan; and Medlmmune.

\section{Availability of data and materials}

Not applicable.

\section{Ethics approval and consent to participate}

This study was approved by the ethics committee (CPP Ile de France II, no. 2008-133, 2012 06-12, 2018 MS1) in agreement with article L.1121-1 of French law.

\section{Consent for publication}

Not applicable.

\section{Competing interests}

This work was made possible by a collaborative research effort between the investigators at CERTIM and the investigators at NanoString. The CERTIM team (led by DD, FG, and KL) contributed samples and clinical information from their patient database, as well as performing the molecular characterizations including PD-L1 IHC, and running the nCounter assay. The NanoString team (SW, XR, PM, and AC) contributed reagents for the gene expression profiling, and performed analysis of the gene expression assays. Both groups contributed to the design of the analyses, interpretation of results, and preparation of the manuscript. RH, HS, RH and TC are full-time employees of Medlmmune and they provided the tumor mutation burden assessment.

\section{Author details}

${ }^{1}$ Team Cancer, Immune Control and Escape, Cordeliers Research Center, UMRS 1138, Institut National de la Santé et de la Recherche Médicale (INSERM), Paris, France. ${ }^{2}$ University Paris Descartes, Paris, France. ${ }^{3}$ Department of Pathology, Hôpital Cochin, AP-HP, Paris, France. ${ }^{4}$ CERTIM, Hôpital Cochin, APHP, Paris, France. ${ }^{5}$ NanoString Technologies, Seattle, WA, USA. ${ }^{6}$ Department of Medical Oncology, Hôpital Cochin, AP-HP, Paris, France. ${ }^{7}$ Team Physiopathologie, cibles et thérapies de la polyarthrite rhumatoide Laboratoire Immunologie et Immunopathologie-Li2P, UMR1125, Université Paris 13, Bobigny, France.

${ }^{8}$ Department of Thoracic Surgery, Hôpital Cochin, AP-HP, Paris, France.

${ }^{9}$ Department of Cutaneous Diseases, Hôpital Cochin, AP-HP, Paris, France.

${ }^{10}$ Genomic platform, INSERM U1016, Institut Cochin, Paris, France. ${ }^{11}$ Oncology Research, Medlmmune, Gaithersburg, MD, USA. ${ }^{12}$ Department of Genetic and Molecular Biology, Hôpital Cochin, AP-HP, 27 rue du Faubourg St Jacques, 75014 Paris, France.

Received: 1 July 2019 Accepted: 15 October 2019

Published online: 04 November 2019

\section{Abbreviations}

CERTIM: Immunomodulatory Therapies Multidisciplinary Study group; CR: complete response; FFPE: formalin-fixed paraffin-embedded; ICl: immune checkpoint inhibitors; IHC: immunohistochemistry; IUO: Investigational Use Only; MSI: microsatellite instability; NSCLC: non-small cell lung cancer; PR: partial response; RECIST: Response Evaluation Criteria in Solid Tumors; RUO: Research Use Only; TIS: tumor inflammation signature; TMB: tumor mutational burden.

\section{Acknowledgements}

We thank Aurélie Boni and Hiromi Sato for their technical assistance.

\section{References}

1. Chen DS, Mellman I. Elements of cancer immunity and the cancerimmune set point. Nature. 2017;541(7637):321-30.

2. Ayers M, Lunceford J, Nebozhyn M, Murphy E, Loboda A, Kaufman DR, et al. IFN-gamma-related mRNA profile predicts clinical response to PD-1

3. Auslander N, Zhang G, Lee JS, Frederick DT, Miao B, Moll T, et al. Robust prediction of response to immune checkpoint blockade therapy in metastatic melanoma. Nat Med. 2018;24(10):1545-9. blockade. J Clin Invest. 2017;127(8):2930-40. 
4. Shin DS, Zaretsky JM, Escuin-Ordinas H, Garcia-Diaz A, Hu-Lieskovan S, Kalbasi A, et al. Primary resistance to PD-1 blockade mediated by JAK1/2 mutations. Cancer Discov. 2017;7(2):188-201.

5. Zaretsky JM, Garcia-Diaz A, Shin DS, Escuin-Ordinas H, Hugo W, HuLieskovan S, et al. Mutations associated with acquired resistance to PD-1 blockade in Melanoma. N Engl J Med. 2016;375(9):819-29.

6. Herbst RS, Soria JC, Kowanetz M, Fine GD, Hamid O, Gordon MS, et al. Predictive correlates of response to the anti-PD-L1 antibody MPDL3280A in cancer patients. Nature. 2014:515(7528):563-7.

7. Rosenberg JE, Hoffman-Censits J, Powles T, van der Heijden MS, Balar AV, Necchi A, et al. Atezolizumab in patients with locally advanced and metastatic urothelial carcinoma who have progressed following treatment with platinum-based chemotherapy: a single-arm, multicentre, phase 2 trial. Lancet. 2016;387(10031):1909-20.

8. Popa S, Church SE, Pekker I, Dowidar N, Sullivan A, Ngouenet C, et al. Validating critical analytical variables of a multiplexed gene expression assay measuring tumor inflammation designed to predict response to anti-PD1 therapy. Poster at ASCO-SITC 2018, Abstract \#203. 2018.

9. Wallden B, Church S, Pekker I, Zimmerman S, Popa S, Sullivan A, et al. Impact of tissue processing and interferents on the reproducibility and robustness of a multi-plex gene expression assay measuring tumor inflammation. Poster at ESMO 2018, Abstract \#4244. 2018.

10. Adam J, Le Stang N, Rouquette I, Cazes A, Badoual C, Pinot-Roussel H, et al. Multicenter harmonization study for PD-L1 IHC testing in non-smallcell lung cancer. Ann Oncol. 2018;29(4):953-8.

11. Biton J, Ouakrim H, Dechartres A, Alifano M, Mansuet-Lupo A, Si H, et al. Impaired tumor-infiltrating T cells in patients with chronic obstructive pulmonary disease impact lung cancer response to PD-1 blockade. Am J Respir Crit Care Med. 2018;198(7):928-40.

12. Danaher P, Warren $S$, Cesano A. Development of gene expression signatures characterizing the tumor-immune interaction. J Clin Oncol. 2018. https://doi.org/10.1200/JCO.2018.36.5_suppl.205.

13. Yu H, Boyle TA, Zhou C, Rimm DL, Hirsch FR. PD-L1 expression in lung cancer. J Thorac Oncol. 2016;11(7):964-75.

14. Rizvi H, Sanchez-Vega F, La K, Chatila W, Jonsson P, Halpenny D, et al. Molecular determinants of response to anti-programmed cell death (PD)-1 and anti-programmed death-ligand 1 (PD-L1) blockade in patients with non-small-cell lung cancer profiled with targeted next-generation sequencing. J Clin Oncol. 2018;36(7):633-41.
15. Carbone DP, Reck M, Paz-Ares L, Creelan B, Horn L, Steins M, et al. First-line nivolumab in stage IV or recurrent non-small-cell lung cancer. N Engl J Med. 2017:376(25):2415-26.

16. Hellmann MD, Ciuleanu TE, Pluzanski A, Lee JS, Otterson GA, AudigierValette $C$, et al. Nivolumab plus ipilimumab in lung cancer with a high tumor mutational burden. N Engl J Med. 2018;378(22):2093-104.

17. Hellmann MD, Nathanson T, Rizvi H, Creelan BC, Sanchez-Vega F, Ahuja A, et al. Genomic features of response to combination immunotherapy in patients with advanced non-small-cell lung cancer. Cancer Cell. 2018;33(5):843.e4-852.e4

18. Cristescu R, Mogg R, Ayers M, Albright A, Murphy E, Yearley J, et al. Pan-tumor genomic biomarkers for PD-1 checkpoint blockade-based immunotherapy. Science. 2018;362(6411):eaar3593.

19. Stenzinger A, Allen JD, Maas J, Stewart MD, Merino DM, Wempe MM, et al. Tumor mutational burden (TMB) standardization initiatives: recommendations for consistent TMB assessment in clinical samples to guide immunotherapy treatment decisions. Genes Chromosomes Cancer. 2019:58(8):578-88.

20. Fehrenbacher L, Spira A, Ballinger M, Kowanetz M, Vansteenkiste J, Mazieres J, et al. Atezolizumab versus docetaxel for patients with previously treated non-small-cell lung cancer (POPLAR): a multicentre, open-label, phase 2 randomised controlled trial. Lancet. 2016;387(10030):1837-46.

21. Prat A, Navarro A, Paré L, Reguart N, Galván P, Pascual T, et al. Immunerelated gene expression profiling after PD-1 blockade in non-small cell lung carcinoma, head and neck squamous cell carcinoma, and melanoma. Cancer Res. 2017;77(13):3540-50.

22. Karachaliou N, Gonzalez-Cao M, Crespo G, Drozdowskyj A, Aldeguer E, Gimenez-Capitan A, et al. Interferon gamma, an important marker of response to immune checkpoint blockade in non-small cell lung cancer and melanoma patients. Ther Adv Med Oncol. 2018;10:1758834017749748.

23. Cesano A, Warren S. Bringing the next generation of immuno-oncology biomarkers to the clinic. Biomedicines. 2018;6(1):14.

\section{Publisher's Note}

Springer Nature remains neutral with regard to jurisdictional claims in published maps and institutional affiliations.
Ready to submit your research? Choose BMC and benefit from:

- fast, convenient online submission

- thorough peer review by experienced researchers in your field

- rapid publication on acceptance

- support for research data, including large and complex data types

- gold Open Access which fosters wider collaboration and increased citations

- maximum visibility for your research: over 100M website views per year

At BMC, research is always in progress.

Learn more biomedcentral.com/submissions 